\title{
Laser-induced acoustic landmine detection with experimental results on buried landmines
}

\author{
J.C. van den Heuvel, F.J.M. van Putten, A.C. van Koersel, H.M.A. Schleijpen \\ TNO Physics and Electronics Laboratory, Oude Waalsdorperweg 63, 2597 AK The Hague, The \\ Netherlands
}

\begin{abstract}
Acoustic landmine detection (ALD) is a technique for the detection of buried landmines including non-metal mines. Since it gives complementary results with GPR or metal detection, sensor fusion of these techniques with acoustic detection would give promising results. Two methods are used for the acoustic excitation of the soil: laser excitation and loudspeaker excitation. A promising concept is using lasers for excitation and monitoring for complete stand-off detection. Results from a field test and laboratory experiments show the feasibility of laser excitation for ALD. In these experiments buried landmine surrogates were measured with ALD using a Nd-YAG laser at $1.06 \mu \mathrm{m}$ for the acoustic generation and a Laser Doppler Vibrometer (LDV) system at $1.54 \mu \mathrm{m}$ for the detection of soil vibrations.

An analysis is given of the experimental results showing the potential and the inherent limitations of the technique. We discuss the relative merits of LDV detection versus microphone detection of the laser-induced acoustic vibration. It was found that the LDV has limitations with respect to microphone detection due to the influence of surface effects that are prominent in LDV but absent in microphone detection.
\end{abstract}

Keywords: landmine detection, buried landmine, non-metal mine, laser Doppler vibrometer

\section{INTRODUCTION}

Reliable and rapid detection of buried land mines - be it antitank mines (ATM) or antipersonnel mines (APM) - was and is a challenging task for the military as well as the civilian community. In this paper the technique of acoustic landmine detection (ALD) is investigated that uses laser excitation as the acoustic source. Another way of acoustic excitation is by means of a loudspeaker. Both excitation techniques have been compared in ref. [1]. Both techniques are valuable in a sensor fusion concept since they provide complementary information about buried objects with respect to GPR or metal detectors.

The ALD technique is a promising detection method, since it does not depend on the metal content of landmine. Thus, metal mines as well as plastic mines (e.g. anti-personnel) can be detected, since this approach is absolutely independent of any metal content within a mine. This is valid for a variety of soil types including soils with ferromagnetic content. With ground-penetrating radar, objects embedded in the soil present a dielectric variation and cause a reflection of the electromagnetic wave. This permits a 3D-mapping of the ground, and in principle allows "fingerprinting" the detected object by multiple reflections in the object. Although ALD with loudspeaker excitation allows only 2D-mapping, in allows a similar type of fingerprinting by analysis of the acoustical resonances of the landmine casing. The loudspeaker excitation gives a good contrast between the buried mine and the surrounding soil at certain frequencies. The technique of laser excitation gives a pulse response that is more difficult to interpret than the loudspeaker excitation but is potentially a faster technique and besides the shape and size information additional depth information could be detected. Laser excitation also seems more suitable for remote detection, since the laser energy can easily be pointed 10 or more meters in front of the detection platform.

In this paper results of field tests and laboratory experiments are presented. It is shown that laser excitation gives a reproducible acoustic excitation at the surface and that the acoustic excitation is coupled into the ground. Using the Laser Doppler Vibrometer (LDV) to measure the acoustic signal at the surface, we found a clear signal of the first peak corresponding to the acoustic surface excitation propagating along the surface. However, the LDV signal after the first peak shows large shot-to-shot variation making detection of buried objects difficult. These shot-to-shot variations are 
caused by surface effects that are prominent in LDV signals but are negligible in microphone signals. This is a severe limitation in the use of an LDV system in combination with laser excitation for ALD.

\section{EXPERIMENTAL SET-UP}

\subsection{Test site}

The experiments were performed in the laboratory and outdoors. The outdoor test consisted of experiments in an outdoor sand area shielded by a large tent. Buried test mines were used. The test mines have signatures close to those of real mines. To simulate the explosives, the devices have been filled with a silicone rubber. Experiments have proven that this is an excellent surrogate of TNT since both substances have the same electromagnetic and thermal characteristics. ${ }^{2}$ In our experiments the vibration issues of the mine surrogates are of crucial importance. Therefore, original mine casings and test mine were used in the experiment in order to allows a comparison between the real casings and the test mines. Figure 1 shows the mines that were used in the sand area.

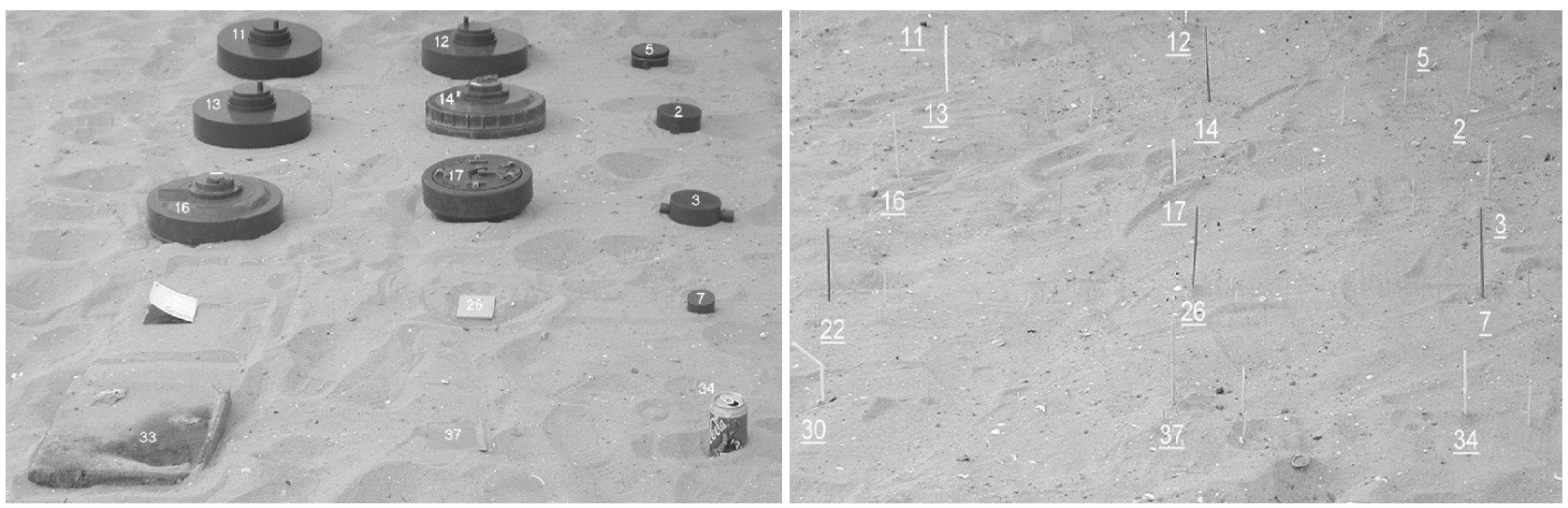

Figure 1: Mines before and after being buried in the sand.

\subsection{Microphones and geophones}

To attempt to measure the acoustic signal in the ground resulting from experiments with the laser and the loudspeaker, three geophones and three microphones were buried in the sand at different depths. In addition, a fourth microphone was mounted $29 \mathrm{~cm}$ above the sand level. Their position and the planned points where the laser was aimed are shown in Figure 2. Microphones are B\&K $1 / 2$ inch pre-polarized condenser microphones (type 4129) in connection with a B\&K preamplifier, Geophones were vertical type SM-6B of Geosource, they have a coil resistance of 375 Ohm and a natural frequency of $4.5 \mathrm{~Hz}$.

Table 1: Sensor Numbering, type, position and sensitivity.

\begin{tabular}{|l|l|l|l|l|}
\hline Sensor & Type & $\begin{array}{l}\text { Position }(\mathrm{x}, \mathrm{y}, \mathrm{z}) \\
\text { in cm }\end{array}$ & $\begin{array}{l}\text { Recorder } \\
\text { Bandwidth }\end{array}$ & Sensitivity \\
\hline M1 & B\&K & $(0,-10,-2)$ & $20 \mathrm{kHz}$ & $-26.2 \mathrm{~dB} \mathrm{V/PA}$ \\
\hline M2 & B\&K & $(0,-20,-5)$ & $20 \mathrm{kHz}$ & $-26.2 \mathrm{~dB} \mathrm{V/PA}$ \\
\hline M3 & B\&K & $(0,-30,-10)$ & $20 \mathrm{kHz}$ & $-24.8 \mathrm{~dB} \mathrm{V/PA}$ \\
\hline M4 & B\&K & $(0,0,-29)$ & $20 \mathrm{kHz}$ & $-24.8 \mathrm{~dB} \mathrm{V/PA}$ \\
\hline G1 & SM6B & $(0,10,-2)$ & $10 \mathrm{kHz}$ & $28.86 \mathrm{~dB} / \mathrm{cm} / \mathrm{s}$ \\
\hline G2 & SM6B & $(0,20,-5)$ & $10 \mathrm{kHz}$ & $28.86 \mathrm{~dB} / \mathrm{cm} / \mathrm{s}$ \\
\hline G3 & SM6B & $(0,30,-10)$ & $10 \mathrm{kHz}$ & $28.86 \mathrm{~dB} / \mathrm{cm} / \mathrm{s}$ \\
\hline
\end{tabular}




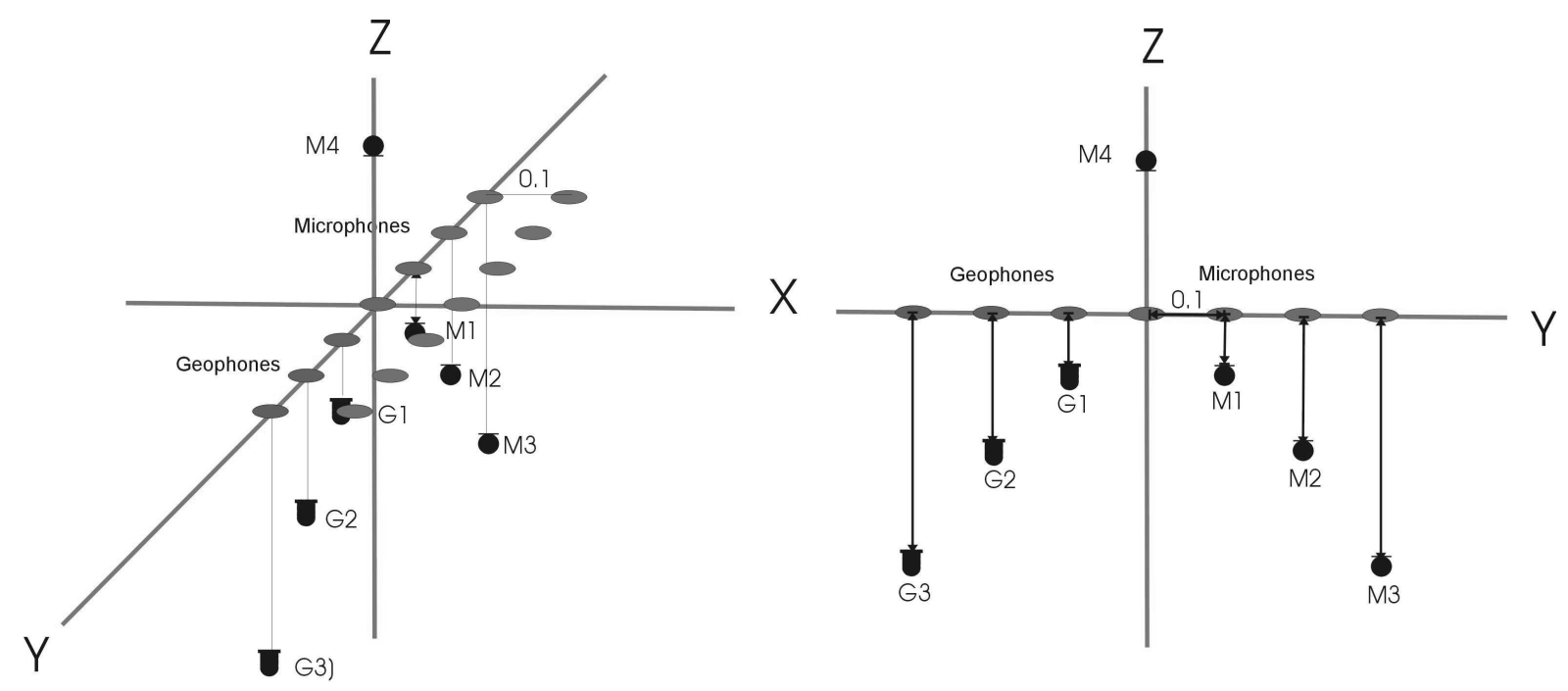

Figure 2: Microphone and geophone positions relative to the origin. Laser spots are indicated by ellipses.

\subsection{Laser excitation}

With laser excitation a Q-switched laser pulse heats a small area of the surface of the soil in a very short time of five nanoseconds. Due to the heating of the soil and the secondary heating of the air in and above the soil, an acoustic pulse is generated that propagates in the soil. The vibrations of the soil's surface are measured with an LDV at a range of several meters. These vibrations are modified due to the presence of a buried mine. ${ }^{4}$ The detection of the mine is based on the change in surface vibration. In first order the acoustic shock wave generated by the laser pulse reflects back from buried objects to the surface; the echo is measured by the LDV.

At present we do not use an integrated system but two separate laser systems: one for excitation and one for laser Doppler vibrometry. During research this is not a disadvantage, however, an operational system has to have an integrated excitation laser and monitoring laser. For the excitation of acoustic shock waves, a Q-switched Nd:YAG laser was used. This laser (Quantel Brilliant) can deliver pulses with an energy up to $350 \mathrm{~mJ}$ with a $20 \mathrm{~Hz}$ rep-rate at $1.064 \mu \mathrm{m}$ (see Figure 3). A variable attenuator and focussing optics resulted in a laser spot of about $1 \times 3 \mathrm{~mm}$ on the sand with an energy of $170 \mathrm{~mJ}$.

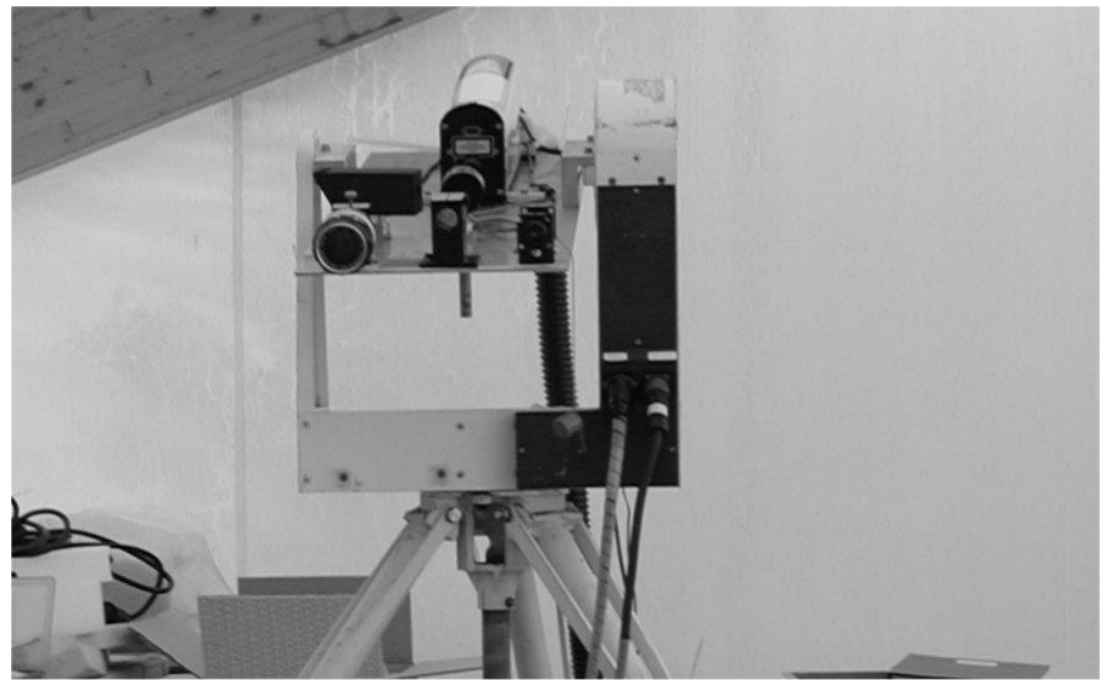

Figure 3: Nd:YAG laser for acoustic excitation. 


\subsection{Laser Doppler vibrometer}

The laser vibrometer used for the experiments was developed at TNO for long range vibration measurements with a 1 Watt $1542 \mathrm{~nm}$ laser source (see Figure 4). Because this high power is not necessary at the short range of a few meters, the power was reduced to about $175 \mathrm{~mW}$.
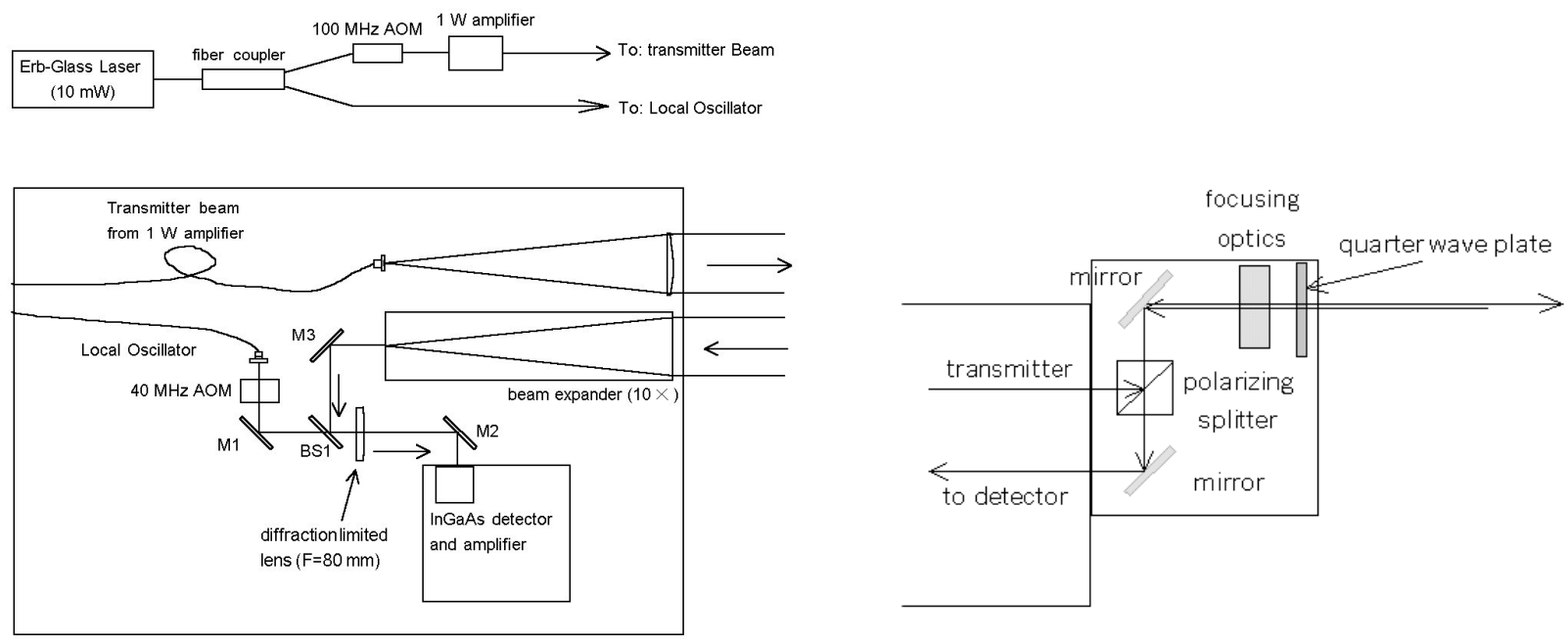

Figure 4: TNO eye-safe laser vibrometer with short range adaptation optics.

Due to the small divergence of the laser and the small field of view of the detector additional optics were placed in front of the system to measure at short distances. Using a polarizing beam-splitter cube and a quarter-wave plate monostatic operation was possible while 2 additional lenses were used to focus at short distances (see fig. Figure 4). The spot size of the laser on the ground was about 1 to $2 \mathrm{~mm}$.

The vibrometer uses 2 Bragg cells $(100 \mathrm{MHz}$ and $40 \mathrm{MHz})$, resulting in a $60 \mathrm{MHz}$ frequency signal at the detector. This signal is mixed down to a frequency of $455 \mathrm{kHz}$. After amplification the signal is fed to a $10 \mathrm{Ms} / \mathrm{s}$ AD card and is stored on the computer for further analysis.

The relative position of the excitation laser spot and the monitoring laser spot has to be optimized. ${ }^{6}$ If the monitoring laser spot is too close to the excitation spot, the received signal is dominated by scattered soil particles that overwhelm the small response of the buried object. However, if the monitoring laser spot is too far away, the sensitivity for the acoustic signal is too low. In order to find the optimum position, the LDV was pointed at a number of spots at various distances from the excitation spot; distances that were used are between 12 and $24 \mathrm{~mm}$. At every position vibration, data from 16 consecutive pulses was collected by a computer.

\section{RESULTS}

\subsection{Excitation of the soil}

The buried microphones are used to measure the acoustic excitation of the soil from the excitation laser. In addition the microphone in air gives information on the acoustic source. Comparison of both microphone signals gives an indication of the coupling of sound into the soil. Figure 5 shows the acoustic signals from the microphone in the air above the laser spot and the microphone below the laser spot buried $2 \mathrm{~cm}$ in the sand. It is clear that there is an acoustic excitation in the ground, however, the sound pressure is reduced considerably. Furthermore, the high sound frequencies are attenuated strongly in the sand. The delay of the microphone in air is due to the longer distance from the sand surface, i.e. $29 \mathrm{~cm}$ versus $2 \mathrm{~cm}$ of the buried microphone. This delay between the signals in the air and in the ground is approximately $0.8 \mathrm{~ms}$, which corresponds quite well with the $27 \mathrm{~cm}$ path length difference and a sound speed of $340 \mathrm{~m} / \mathrm{s}$. 

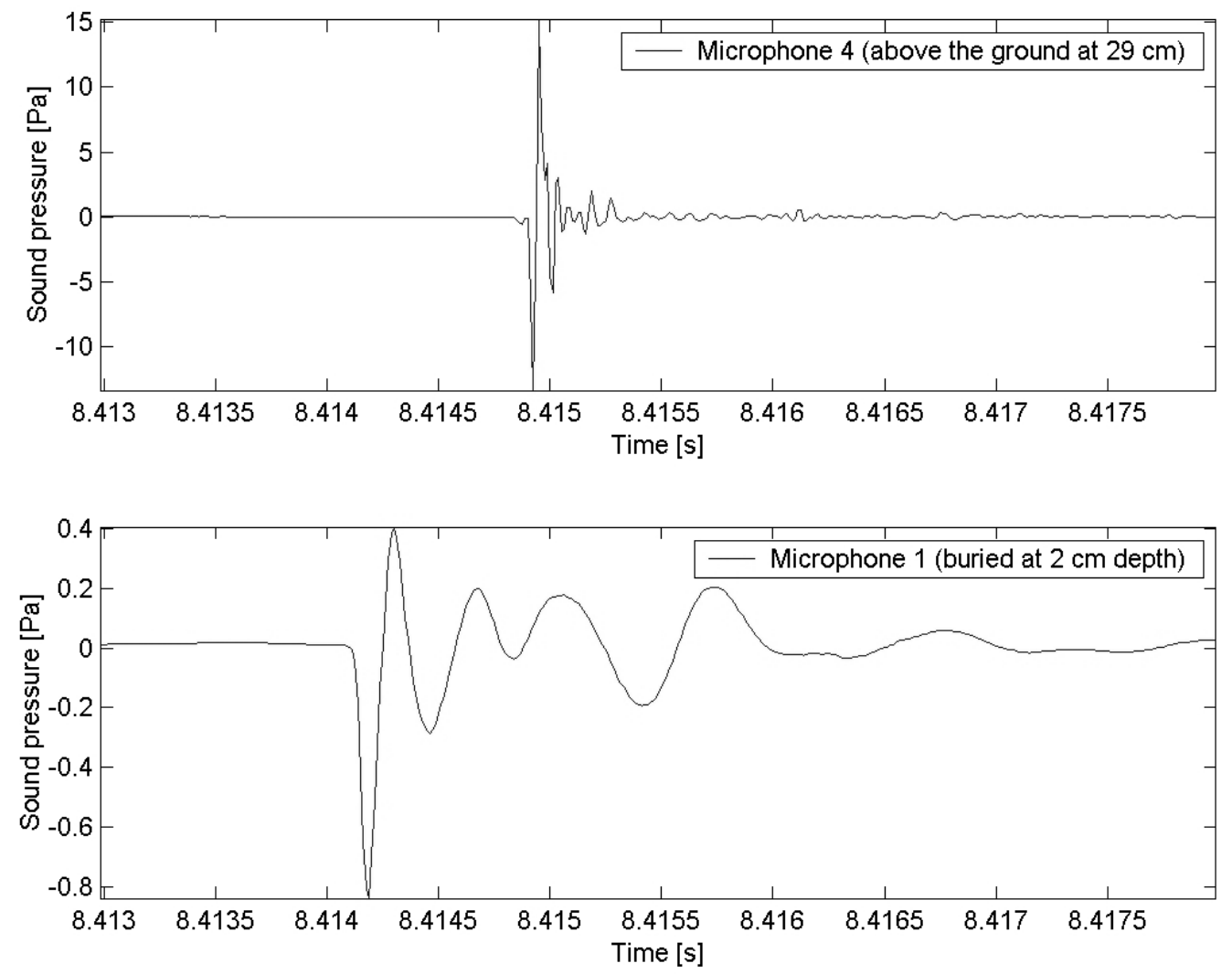

Figure 5: Top graph is the microphone in the air, bottom graph is the microphone buried at $2 \mathrm{~cm}$ depth. The laser is aimed at a spot on the ground directly above the buried microphone. The graph shows $5 \mathrm{~ms}$ of recorded data with one pulse

The shot-to-shot variation of the acoustic signal is quite small. Figure 6 shows the acoustic signals of five consecutive laser shots. This means that the excitation of the sand is quite reproducible, which is a requirement for the detection of buried objects by the LDV system. 

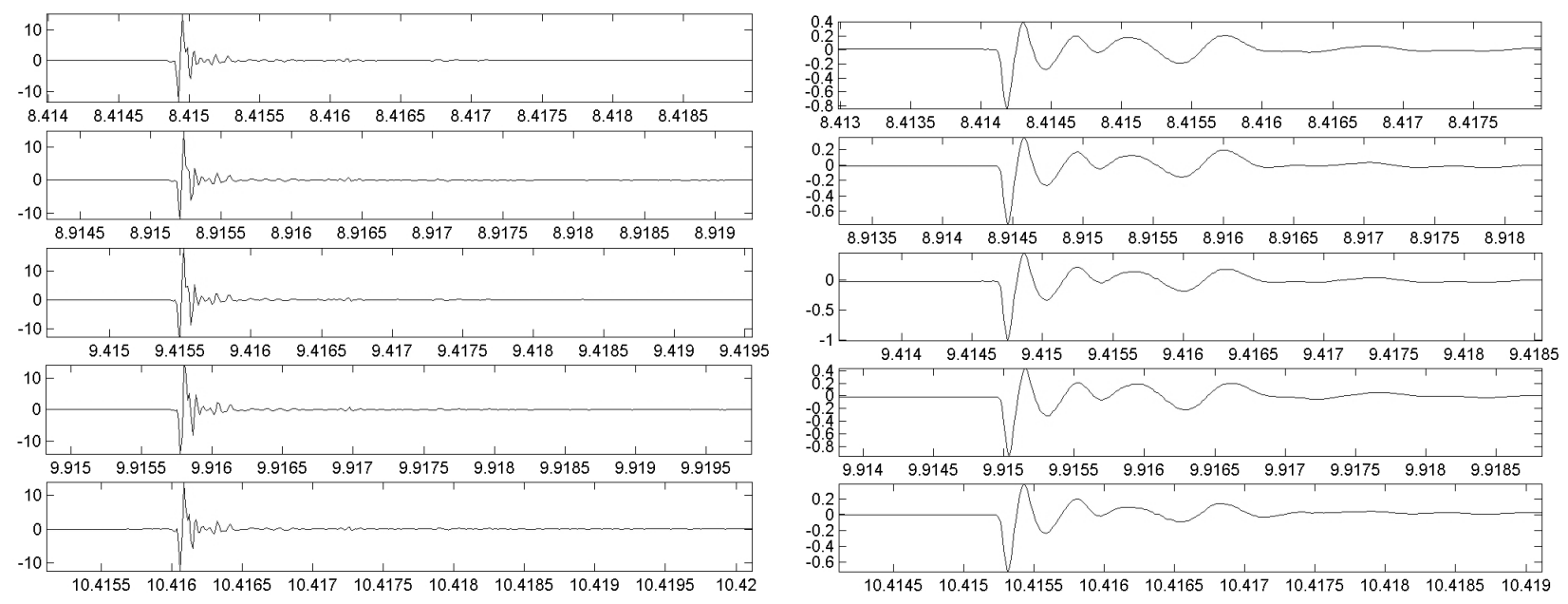

Figure 6: Five consecutive acoustic signals of the microphone in air (left) and buried in the sand (right).

\subsection{Laser Doppler vibrometer}

The acoustic signal results in a frequency modulation of the $455 \mathrm{kHz}$ high frequency carrier as a result of the Doppler frequency shift $2 v / \lambda$, where $v$ is the velocity and $\lambda$ is the wavelength of the laser. MATLAB was used to demodulate the high frequency signal in order to obtain the instantaneous velocity as a function of time.

The Nd:YAG laser was aimed at the center of mine 11 of which the center was $1 \mathrm{~cm}$ deep buried in the sand. Mine 11 is a test mine simulating the anti-tank mine TM62P. As a reference the Nd:YAG laser was also aimed at a position without a buried object. The demodulated signals are shown in Figure 7; the figures on the left and on the right are with and without mine, respectively. About $200 \mu \mathrm{s}$ after the data acquisition $\mathrm{AD}$ card is triggered the laser pulse hits the sand. A clear response is visible due to the surface excitation. Clearly, the LDV measures the acoustic wave created by the laser pulse (first peaks). After about $50 \mu$ s another signal is measured as the result of the reflection of the acoustic wave from the mine top. ${ }^{3}$ This delay corresponds quite well with the depth of $1 \mathrm{~cm}$ and an expected sound velocity of about $340 \mathrm{~m} / \mathrm{s} .{ }^{5}$ If there is no mine beneath the laser spots only the first signal peaks are measured by the LDV.
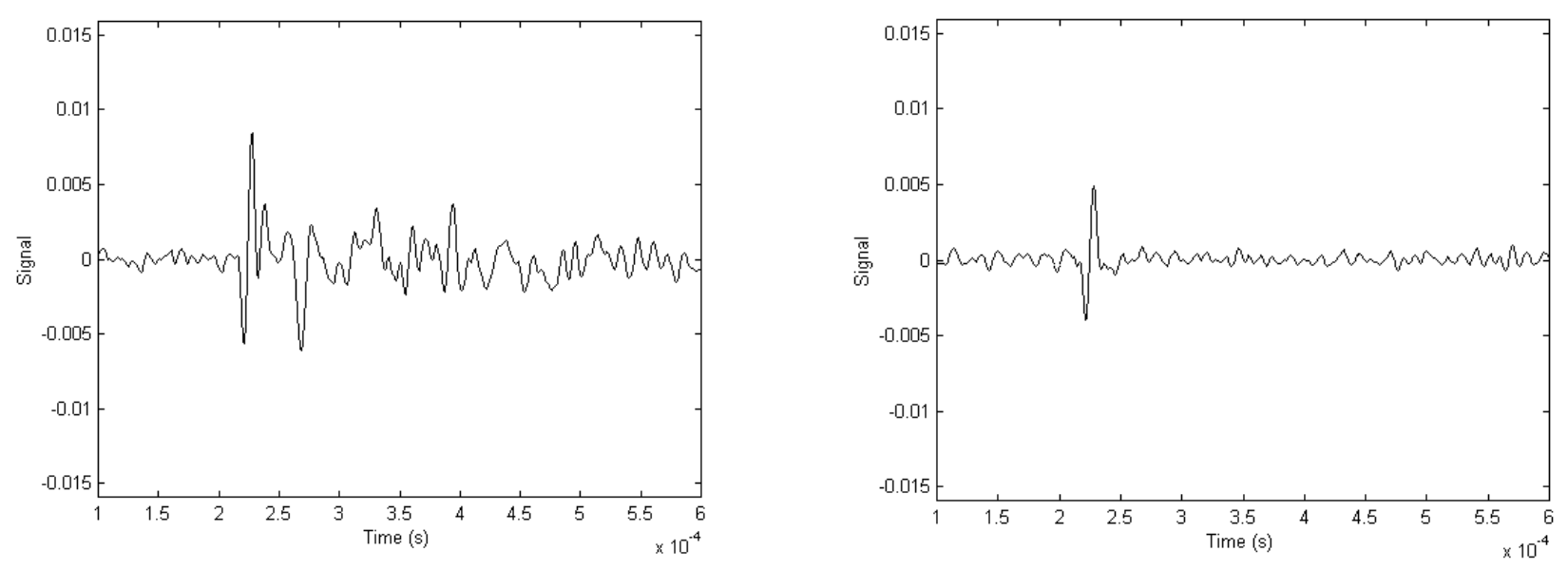

Figure 7: reflected vibration signal from mine 11 (TM62P) at $1 \mathrm{~cm}$ depth (left) and signal without mine (right).

The response of the soil shows large variations from shot to shot, both in the first (surface) peak as in the reflection from the test mine. These variations make the interpretation of the data difficult. It is not clear yet whether it is possible to extract more from the data than the approximate depth of a buried object. In addition, we have to be cautious in attributing the second peak as an echo of the buried mine. As shown previously in Figure 5, the high frequencies are 
attenuated strongly in the sand. Since for a significant echo in a time scale of $50 \mu \mathrm{m}$ we need high frequencies propagating in the sand, it is not clear whether the interpretation of an echo due to the buried mine is reliable.

\subsection{Influence of laser parameters}

The relative position of the excitation laser spot and the probe laser spot was investigated. It is not possible to have the spot of the probe laser at the same position as the spot of the excitation laser, since the excitation laser disturbs the probe laser. Figure 8 shows the LDV signal when the spots of the two laser beam overlap. The large peaks in the signal are caused by fast moving particles (sand, dust) that are projected from the surface due to the laser excitation.

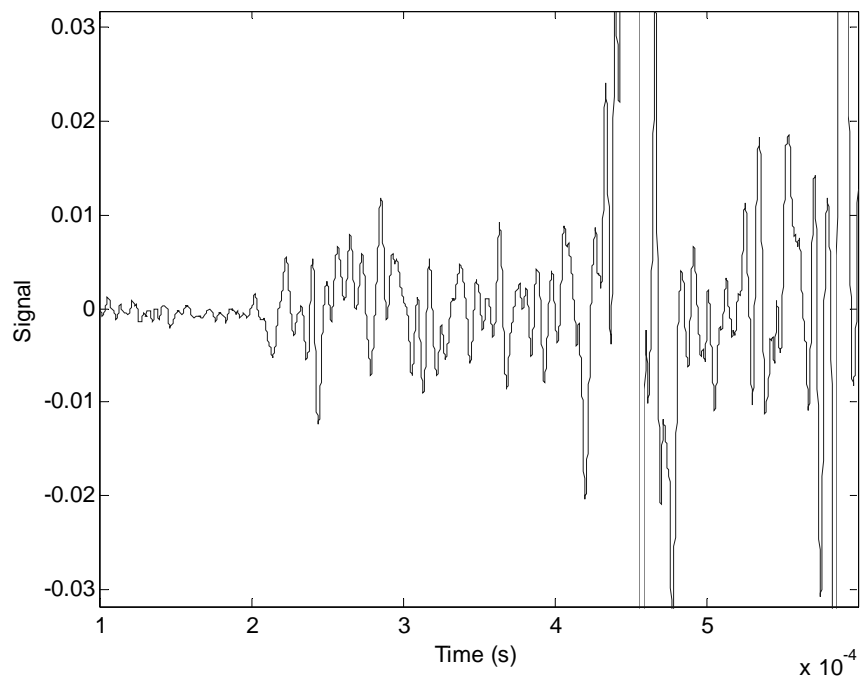

Figure 8: Disturbance of the LDV signal by the laser excitation.

For a reliable signal, it is required to separate the two laser spots. Figure 9 shows the LDV signal for various separations between probe and excitation laser spots in the situation without a buried object. The first peak is always at the same position and can be attributed to electrical interference of the excitation laser, which was located near the LDV system in these laboratory experiments. In addition, a different excitation laser was used from that in the field experiment with different electronics for the triggering. For the second peak, it is clear that an increased separation causes an increased delay. This second peak is the acoustic wave that propagates along the surface. Figure 10 shows the delay of the LDV signal peak as a function of distance between laser excitation spot and LDV probe spot. The slope of the straight line that connects the points corresponds to a velocity of $340 \mathrm{~m} / \mathrm{s}$, which corresponds to the speed of sound. 


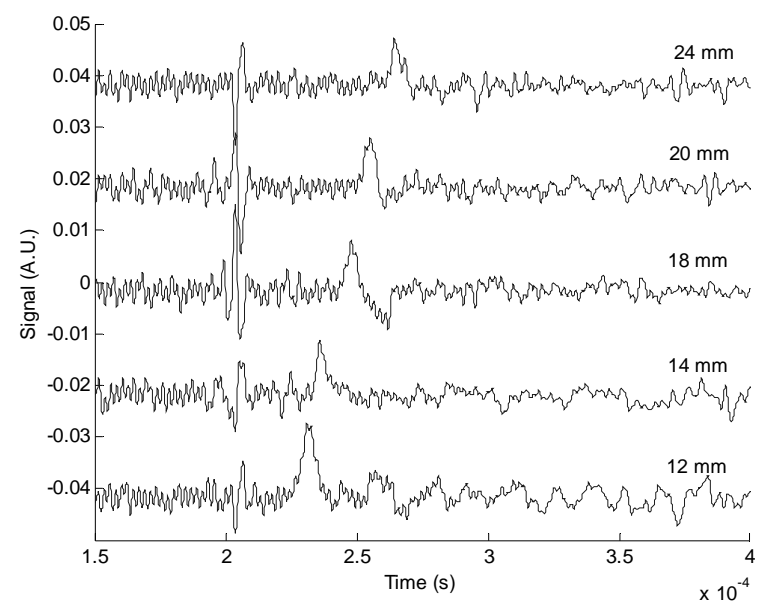

Figure 9: LDV signal for various separations between probe and excitation laser spots.

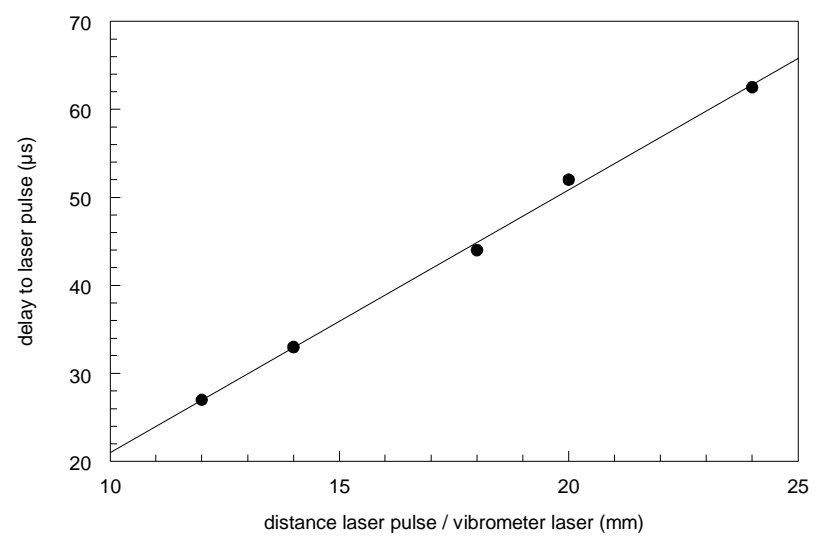

Figure 10: Delay of LDV signal as a function of spot separation.

Unfortunately, the shot-to-shot variation of the LDV signal is quite large and increases when a buried object is present. The LDV signals of Figure 10 were selected from many shots. Approximately $50 \%$ of the shots showed the peak in the LDV at the expected position. The other shots showed large disturbances in the LDV signal.

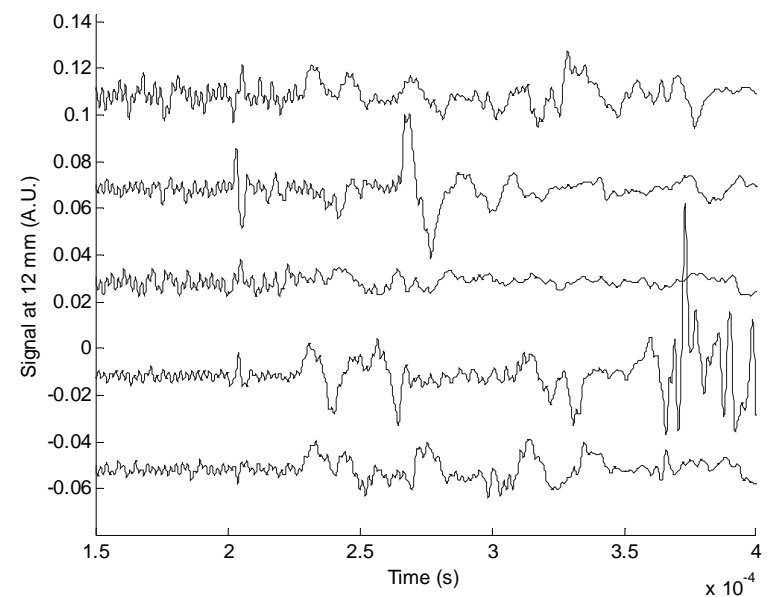

Figure 11: Shot-to-shot variation of the LDV signal WITH buried object.

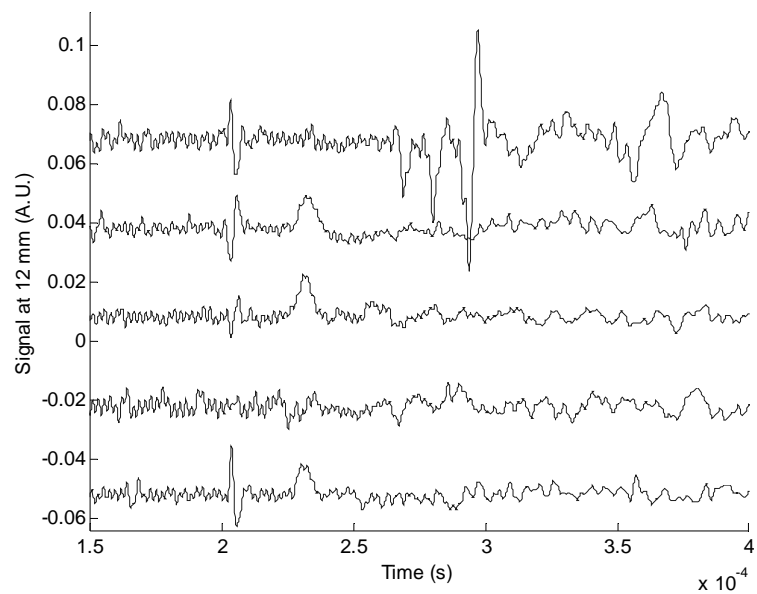

Figure 12: Shot-to-shot variation of the LDV signal WITHOUT buried object.

Figure 11 and Figure 12 show the shot-to-shot variation for the situation with and without a buried object. Both figures show results with the excitation and the probe laser spot at a separation of $12 \mathrm{~mm}$. For some shots with a buried object, it is possible to see a peak at the expected position corresponding to the echo from the buried object. A discussion of these large variations is given in the next section.

\section{DISCUSSION}

\subsection{Surface effects}

The LDV system measures the velocity of the surface at the spot of the probe laser by determining the frequency shift with an FM receiver. Since we know that the Doppler shift is $2 v / \lambda$, the signal before FM demodulation is given by 


$$
s(t)=A(t) \sin \left(2 \pi f_{C} t+2 \pi \frac{2 v}{\lambda} t\right)=A(t) \sin \left(2 \pi f_{C} t+2 \pi \phi(t)\right),
$$

where $A(t)$ is the amplitude, $\phi(t)$ is the time dependent phase, and $f_{C}$ is the carrier frequency. FM demodulation is the time differentiation of the phase (ignoring the carrier frequency). It is clear from this equation that there is a non-linear relation between two laser-light reflecting points, i.e. the FM demodulation of $s_{I}(t)+s_{2}(t)$ is not the time differentiation of $\phi_{1}(t)+\phi_{2}(t)$. This means in practice that areas with different velocities within the laser spot lead to a distorted acoustic signal. Therefore, the laser spot is focused to obtain a clear acoustic signal. However, the consequence is that the LDV signal is very sensitive to local effects and only shows the vibration of a very small surface spot and not the average of the surface vibration as in the microphone experiments that measure pressure variations caused by the entire vibrating surface.

Another surface effect that is typical of laser sensing systems is the speckle effect. Due to the high coherence of the laser light the contribution of surface scatterers is added coherently. In imaging systems this leads to granular images, i.e. images with small grains of light and dark spots (speckle). In an LDV system this speckle effect is the cause of extra noise.

Finally, the LDV system is sensitive to fast moving debris from the laser excitation. This effect is absent in microphone measurements.

\subsection{LDV versus microphone detection}

Detection of the acoustic signal from a buried using an LDV instead of a microphone shows the prospect of stand-off detection using laser excitation for the generation of the acoustic signal and an LDV for detection of the acoustic signature. An additional anticipated advantage of an LDV over a microphone is that an LDV measures the vibration directly at the surface level while the microphone measures in air above the ground. Therefore, the LDV is expected to be more sensitive and to show fewer disturbances from other acoustic noise sources.

However, we have shown that using an LDV gives large shot-to-shot variation, which can be attributed to surface effects. It is not clear how these effects can be reduced in order to make the combination laser excitation and LDV into a reliable system for acoustic landmine detection.

\section{CONCLUSIONS}

A field test and laboratory experiments of acoustic landmine detection (ALD) were performed. Laser excitation was used for the generation of the acoustic signal and Laser Doppler vibrometry was used to detect the acoustic signature of the buried surrogate mine.

It was found that the acoustic signal generated by the laser excitation has a high reproducibility from shot to shot as was registered by a microphone above the excitation. A buried microphone showed that the acoustic signal is coupled into the ground with a high reproducibility. The LDV sensor showed consistent results for the acoustic signal from the surface. However, there was a large shot-to-shot variation. This variation between different shots from the excitation laser made analysis of the acoustic signal from a buried mine unreliable.

The shot-to-shot variation of the LDV system is attributed to surface effects that are dominant in an LDV system but are absent for microphones. These surface effects are local variations due to the small laser spot, speckle effects, and debris from the laser excitation that pass the LDV probe beam. These surface effects should be minimized in order to obtain a reliable ALD detection system.

\section{REFERENCES}

1. J.C. van den Heuvel, V.Klein, P. Lutzmann, F.J.M. van Putten,M. Hebel, and H.M.A. Schleijpen, "Sound wave and laser excitation for acousto-optical landmine detection", in Proc. SPIE, Detection and Remediation Technologies for Mines and Minelike Targets VIII, Orlando (FL), USA, April 2003.

2. W. de Jong, H. A. Lensen, and Y. H. L. Janssen, "Sophisticated test facility to detect land mines," in Proc. SPIE Vol. 3710, Detection and Remediation Technologies for Mines and Minelike Targets IV, A. C. Dubey and J. F. Harvey, eds., pp. 1409-1419, (Orlando (FL), USA), Apr. 1999. 
3. W. Li, C.A. DiMarzio and S.W. McKnight, "Laser-induced Acoustic Detection of Shallow-Buried Objects", Proc. SPIE Vol.3752, July 1999.

4. S.W. McKnight et.al., "Laser-induced acoustic generation for buried object detection", Proc. SPIE Vol. 4038 (April 2000).

5. S.W. McKnight, J. Stott, C.A. DiMarzio, R Cleveland, R. Roy, "Laser-induced acoustic imaging of buried land mines: experiment and modeling", SPIE vol 4394, pp. 627-633.

6. Charles A. DiMarzio, et. al., Toward a Laser-Based, "Non-Contact Acoustic Landmine Imager"; Proc. SPIE Vol. 4038 (April 2000). 